\title{
EDITORIAL
}

\section{The NIH consensus criteria for chronic graft-versus-host disease: far more than just another classification}

Leukemia (2009) 23, 1-2; doi:10.1038/leu.2008.277

This invited Editorial Leukemia is addressed at the paper by Cho et al. ${ }^{1}$ published in this issue of Leukemia on the feasibility of using the $\mathrm{NIH}$ consensus criteria for chronic graft-versus-host disease.

Chronic graft-versus-host disease (cGVHD), a multi-organ disorder, is the leading cause of late nonrelapse mortality after hematopoietic stem cell transplant. It has been known for many years that although the disease usually manifests itself more than 100 days after transplant, earlier disease onset could occur. More importantly, clinical syndromes with features of typical acute GVHD are increasingly recognized beyond 100 days after hematopoietic stem cell transplant, especially in recent years with the development of the reduced-intensity conditioning regimen. ${ }^{2}$ In addition, patients with acute GVHD may progress to developing CGVHD with symptoms of both acute GVHD and cGVHD. For many years, we have used a grading system, developed by the Seattle group, ${ }^{3}$ of limited versus extensive GVHD. This study was designed to identify patients needing systemic immune suppression, but it does not capture the severity of individual organ involvement. Although other grading schemes have been proposed (review in Lee $\mathrm{SJ}^{4}$ ) to predict survival following cGVHD, all lack consistent scoring and assessment of each organ involved in determining the overall severity of the disease. Recognizing these limitations, a group of experts led by Dr Pavletic at the National Institutes of Health (NIH) met in 2004 for a consensus conference on cGVHD. As all participants agreed that it was urgently necessary to get rid of the formal definition of CGVHD (any GVHD beyond day 100), the diagnosis and staging working group of the $\mathrm{NIH}$ Consensus Development Project on $\mathrm{CGVHD}^{5}$ proposed standard criteria for diagnosis (Table 1), organ scoring and global assessment of cGVHD severity (see reference 5 for details on how to score organ severity and to assign a severity grade).

To assess the applicability of NIH consensus criteria for CGVHD, Cho et al. in this issue of Leukemia ${ }^{1}$ studied 211 patients who

Table 1 NIH criteria for acute and chronic GVHD

\begin{tabular}{llll}
\hline Category & $\begin{array}{l}\text { Time of } \\
\text { symptoms }\end{array}$ & $\begin{array}{l}\text { Presence } \\
\text { of acute } \\
\text { GVHD } \\
\text { features }\end{array}$ & $\begin{array}{l}\text { Presence } \\
\text { of chronic } \\
\text { GVHD } \\
\text { features }\end{array}$ \\
\hline $\begin{array}{l}\text { Acute GVHD } \\
\text { Classic acute }\end{array}$ & $<100$ days & Yes & No \\
$\begin{array}{l}\text { Persistent } \\
\text { Recurrent } \\
\text { Late onset acute }\end{array}$ & Yes & No \\
$\begin{array}{l}\text { Chronic GVHD } \\
\text { Classic chronic } \\
\text { Overlap syndrome }\end{array}$ & No time limits & No & \\
\hline
\end{tabular}

developed GVHD more than 100 days after allogeneic transplantation and who were reclassified using the NIH criteria. Classifications were late acute GVHD (21\%), overlap syndrome $(30 \%)$ and classic cGVHD (49\%). Classic cGVHD and overlap syndrome patients $(n=167)$ were graded using both the revised Seattle criteria and $\mathrm{NIH}$ global scoring. This is the largest study addressing the critical point of what is left with cGVHD using stringent NIH disease criteria. Two other studies have been published on the same subject: one by the Nashville group involving 110 patients $^{6}$ and the other by the Minneapolis group involving 54 patients. ${ }^{7}$ Classifications in both studies were late acute GVHD (36 and $15 \%$ ), overlap syndrome (26 and $28 \%$ ) and classic cGVHD (37 and $57 \%$ ) (estimates from references 6 and 7, respectively). Despite not being based on the same patient numbers and including different patient, disease and transplant characteristics, one can thus reasonably assume that approximately $20 \%$ of patients formally classified as 'chronic' GVHD using the Seattle day 100 landmark could in fact be considered as having features of an acute inflammatory disease. Is that purely semantic? I do not believe so. This means that all estimates currently published in the literature underestimate acute GVHD incidence and overestimate that of cGVHD. This is not of major importance if you are aware of this caveat; however, it is of importance whether you want to use these incidences to calculate the power of a clinical trial or whether you want to search for a statistical link between acute GVHD and cGVHD with relapse (GVL effect), for example.

An intriguing result in Dr Cho's study is the lack of difference in GVHD-specific survival between patients with late acute GVHD and those with classical cGVHD or with the overlap syndrome. Although not using the same statistical tool, this is in sharp contrast with the results of two other studies, both of which showed a worse prognosis in patients developing late acute GVHD (that clearly fits in better with my own clinical practice). However, in the large Korean group study, the pattern of acute GVHD onset was significantly different with respect to GVHD-specific survival with worse survival for patients with recurrent late acute GVHD.

Among patients with overlap syndrome and classic cGVHD, Cho et al. ${ }^{1}$ multivariate analysis showed that both the NIH severity index and the Seattle group classification were useful in predicting survival and discontinuation of immunosuppressive therapy. Using other methods, the Nashville and the Minneapolis groups also suggest that the new classification of the severity of the disease (mild/moderate/severe) could be useful. However, from the three studies available so far, ${ }^{1-3}$ it is not yet clear whether this new classification will be a better discriminator of disease severity than the classical (limited/extensive) Seattle classification, as the mild plus moderate categories seem to need to be collapsed to reach significant differences as compared with the severe category.

In summary, although it might be highly surprising that it has taken decades of allogeneic transplantation, we do now have in hand a powerful definition of what is 'acute' and what is 'chronic' GVHD, an absolute premise for clinical trial design and for reporting clinical results. Concerning the NIH severity index, my own biased belief is that only the two ongoing 
randomized trials (both in the US and in Europe) will be able to test its usefulness in predicting the cGVHD-specific survival.

\section{Acknowledgements}

I thank Dr M Arora and Prof D Weisdorf for sharing with me the data of their in press study. ${ }^{7}$

G Socié ${ }^{1,2,3}$

${ }^{1}$ Service d'Hématologie Greffe, Inserm, U728, Paris, France, ${ }^{2}$ Université Paris VII, IUH, Paris, France and

${ }^{3}$ AP-HP, Hôpital Saint-Louis, Service d'Hématologie Greffe, Paris, France E-mail: gerard.socie@sls.aphp.fr

\section{References}

1 Cho BK, Min CK, Eom KS, Kim YJ, Kim HJ, Lee S et al. Feasibility of $\mathrm{NIH}$ consensus criteria for chronic graft-versus-host disease. Leukemia 2008; 23: 78-84 (this issue).
2 Mielcarek M, Martin PJ, Leisenring W, Flowers ME, Maloney DG, Sandmaier BM et al. Graft-versus-host disease after non myeloablative versus conventional hematopoietic stem cell transplantation. Blood 2003; 102: 756-762.

3 Shulman HM, Sullivan KM, Weiden PL, McDonald GB, Striker GE, Sale GE et al. Chronic graft-versus-host syndrome in man. A longterm clinico-pathologic study of 20 Seattle patients. Am J Med 1980; 69: 204-217.

4 Lee SJ, Vogelsang G, Flowers ME. Chronic graft-versus-host disease. Biol Blood Marrow Transplant 2003; 9: 215-233.

5 Filipovich AH, Weisdorf D, Pavletic S, Socie G, Wingard JR, Lee SJ et al. National Institutes of Health consensus development project on criteria for clinical trials in chronic graft-versus-host disease: I. Diagnosis and staging working group report. Biol Blood Marrow Transplant 2005; 11: 945-956.

6 Jagasia M, Giglia J, Chinratanalab W, Dixon S, Chen H, Frangoul H et al. Incidence and outcome of chronic graft-versus-host disease using national institutes of health consensus criteria. Biol Blood Marrow Transplant 2007; 13: 1207-1215.

7 Arora M, Nagaraj S, Witte J, DeFor TE, MacMillan ML, Burns LJ et al. New classification of chronic graft versus host disease: added clarity from the consensus diagnoses. Bone Marrow Transplantation 2008; e-pub ahead of print 15 September 2008; doi:10.1038/bmt.2008.305. 